\title{
Absolute pressure measurements on a nanosecond time scale using surface plasmons
}

\author{
A. Schilling, O. Yavaş, J. Bischof, J. Boneberg, and P. Leiderer ${ }^{a)}$ \\ Universität Konstanz, Fakultät für Physik, Postfach 5560 M676, D-78434 Konstanz, Germany
}

(Received 9 September 1996; accepted for publication 30 October 1996)

Transient acoustic waves generated by laser-induced bubble formation at a liquid-solid interface are sensitively monitored using optically excited surface plasmons. This method enables the detection of both the compressive and tensile waves with high accuracy as demonstrated for the propagation and reflection of acoustic pulses at a quartz-water interface. Unique advantages of this new technique are the high sensitivity of 0.1-0.2 MPa that could be achieved for absolute pressure measurements on a nanosecond time scale and its ability to probe exact pulse profiles due to the localized probe depth of surface plasmons. (C) 1996 American Institute of Physics.

[S0003-6951(96)03353-0]

The accurate measurement of absolute pressure amplitudes on the nanosecond time scale is an essential requirement for a better understanding of various technological processes. For example, in shock wave lithotripsy the amplitude of the applied shock wave has to be sufficiently high to induce the fragmentation of urinary or biliary stones. On the other hand, it has to be lower than a certain threshold level as to avoid damage to the surrounding tissue. ${ }^{1}$ In laser cleaning, a recently introduced method to remove submicron particles and organic film-type contaminants from solid surfaces which motivated the work presented here, the high removal efficiency is attributed to the generation of high-intensity acoustic pulses by the laser irradiation. ${ }^{2-4}$ Recent studies using photoacoustic probe beam deflection and piezoelectric transducer methods have indeed confirmed that the acoustic wave intensity is considerably increased due to laser-induced explosive bubble nucleation. ${ }^{5-8}$

However, the test methods used so far have several drawbacks: the probe beam deflection method is limited in its temporal resolution due to the finite beam waist of the probe beam, and for absolute pressure measurements it is required to estimate the pressure gradient and pulse width theoretically. ${ }^{9}$ Similarly, the piezoelectric transducer also provides a limited temporal resolution due to the lack of bandwidth, and is not able to resolve the temporal profile of the acoustic wave due to a resonance effect within the transducer caused by the acoustic impedance mismatch between transducer and surrounding liquid. Consequently, previous studies could not provide a quantitative analysis of the transient acoustic waves associated with the laser-cleaning process.

Here, we present a new approach for the absolute measurement of acoustic wave amplitudes with nanosecond time resolution. It utilizes surface plasmons, which are known to be a sensitive optical tool to probe metal-dielectric interfaces. ${ }^{10-12}$ The sensitive dependence of the surface plasmon resonance upon changes in the refractive index of the water layer taking place within a few hundred nanometers adjacent to the interface, was used to monitor the pressure changes. The experimental setup is depicted in Fig. 1. A thin silver film of $53 \mathrm{~nm}$ was evaporated in high vacuum onto the

${ }^{a)}$ Electronic mail: Paul.Leiderer@uni-konstanz.de base of a heavy flint glass prism $(n=1.7494$ for $\lambda=632.8$ $\mathrm{nm}^{13}$ ). A $2 \mathrm{~nm}$ thick chromium interlayer was used to increase the adhesion between the silver film and the glass prism. The prism with the silver film was mounted on a cuvette filled with distilled water. Surface plasmons were excited optically at the silver-water interface of the prism via attenuated total reflection (ATR) in the Kretschmann configuration using a $5 \mathrm{~mW} \mathrm{He}-\mathrm{Ne}$ probe laser. ${ }^{14}$ The main advantages of the surface plasmon technique over conventional techniques are that the pressure measurement is inherently absolute and that a high temporal resolution (better than $1 \mathrm{~ns}$ ) is achieved, which is in our case limited only by the electronics.

First, static measurements have been performed in order to check the agreement between the experimentally observed and theoretically calculated plasmon resonance shift as a function of pressure. Therefore, we externally applied static pressures up to $0.7 \mathrm{MPa}$ and measured the surface plasmon resonance shift by recording the reflectivity change, in this case on the left wing of the resonance, with a photodiode. The calculations were done with an extensively tested com-

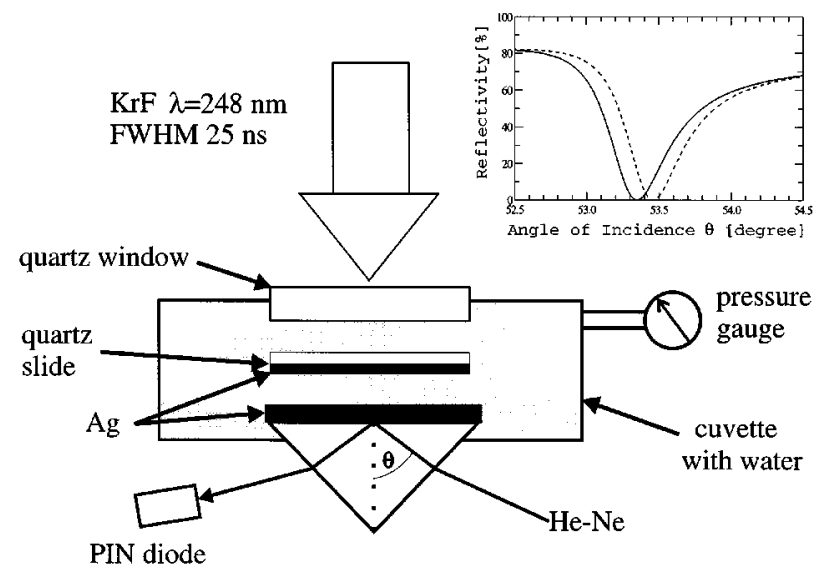

FIG. 1. Experimental setup: an acoustic pulse is generated by pulsed laser heating of the silver film on the quartz slide. The pulse propagates toward the prism and causes a resonance shift upon arrival at the water-silver interface. The shift is detected with a fast photodiode. The inset shows the surface plasmon resonance shift due to an increase in the refractive index of water with pressure. Solid and dotted lines indicate the calculated resonance at ambient pressure and $12 \mathrm{MPa}$, respectively. 


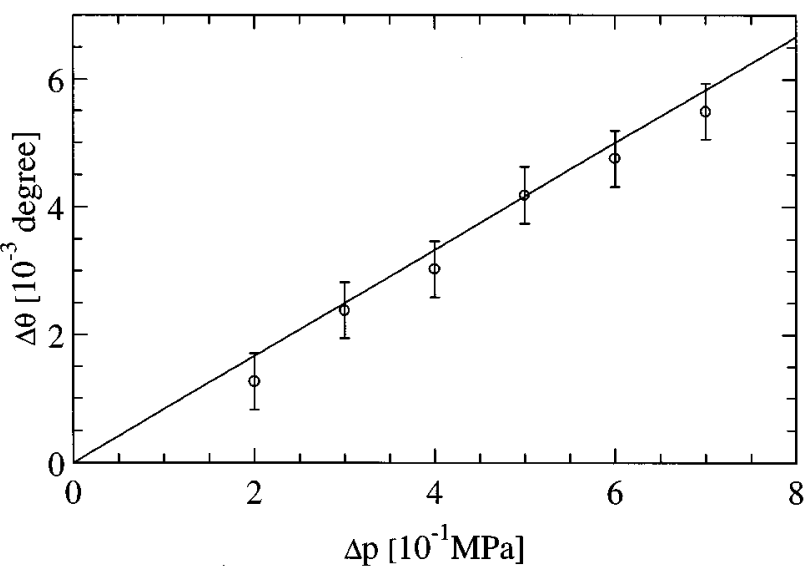

FIG. 2. Static calibration curve for the plasmon resonance shift as a function of water pressure. The data points are measured, the line is calculated. The error bars represent typical deviations which occurred during repeated measurements.

puter program which uses a transfer matrix method and determines reflectivity as a function of the angle of incidence. The refractive index of water as a function of pressure for $\lambda=632.8 \mathrm{~nm}$ was obtained from Schiebener et al $^{15}$

The actual dynamic measurements have been conducted using transient acoustic pulses. As shown in previous experiments, an acoustic pulse is generated when rapid bubble growth sets in following pulsed laser heating of a liquidsolid interface. ${ }^{5-8}$ For this purpose a thin quartz slide, onto which $20 \mathrm{~nm}$ chromium and $80 \mathrm{~nm}$ silver were evaporated, was placed in front of the prism and heated from the rear side by a $\mathrm{KrF}$ excimer laser pulse $(\lambda=248 \mathrm{~nm}$, full width at half maximum $(\mathrm{FWHM})=25 \mathrm{~ns}$ ) (see Fig. 1). The acoustic pulse generated by the explosive bubble growth at the silver-water interface of the quartz slide propagates toward the prism. At the silver-water interface of the prism, the pulse is detected by recording the transient shift of the plasmon resonance due to the pressure-induced change of the dielectric function of the water layer adjacent to the silver film. A positive pressure amplitude increases the refractive index and causes a resonance shift to greater angles. Time-resolved reflectivity measurements were done at a fixed angle on the left wing of the plasmon resonance using a fast $p-i-n$ photodiode (rise time $\leqslant 1 \mathrm{~ns}$ ). The signals were amplified with an ac-coupled 1 $\mathrm{GHz}$ bandwidth amplifier and recorded with a $500 \mathrm{MHz}$ digitizing storage oscilloscope.

The result of the static experiment, where the surface plasmon resonance shift was measured as a function of the static water pressure, is shown in Fig. 2. The resonance shifts, which are on the order of $10^{-3^{\circ}}$, correspond to refractive index changes of about $10^{-5}-10^{-4}$. The data points with error bars are measured, whereas the straight line is computed. The calculation includes a small correction of the plasmon shift due to an increase of the index of refraction with pressure for the heavy flint glass prism. Taking into account that the calculation does not include any fit parameter, the agreement between calculation and experiment is quite satisfactory.

Typical transient signals for two different laser fluences are shown in Fig. 3. At $t=0 \mu$ s the excimer laser pulse heats
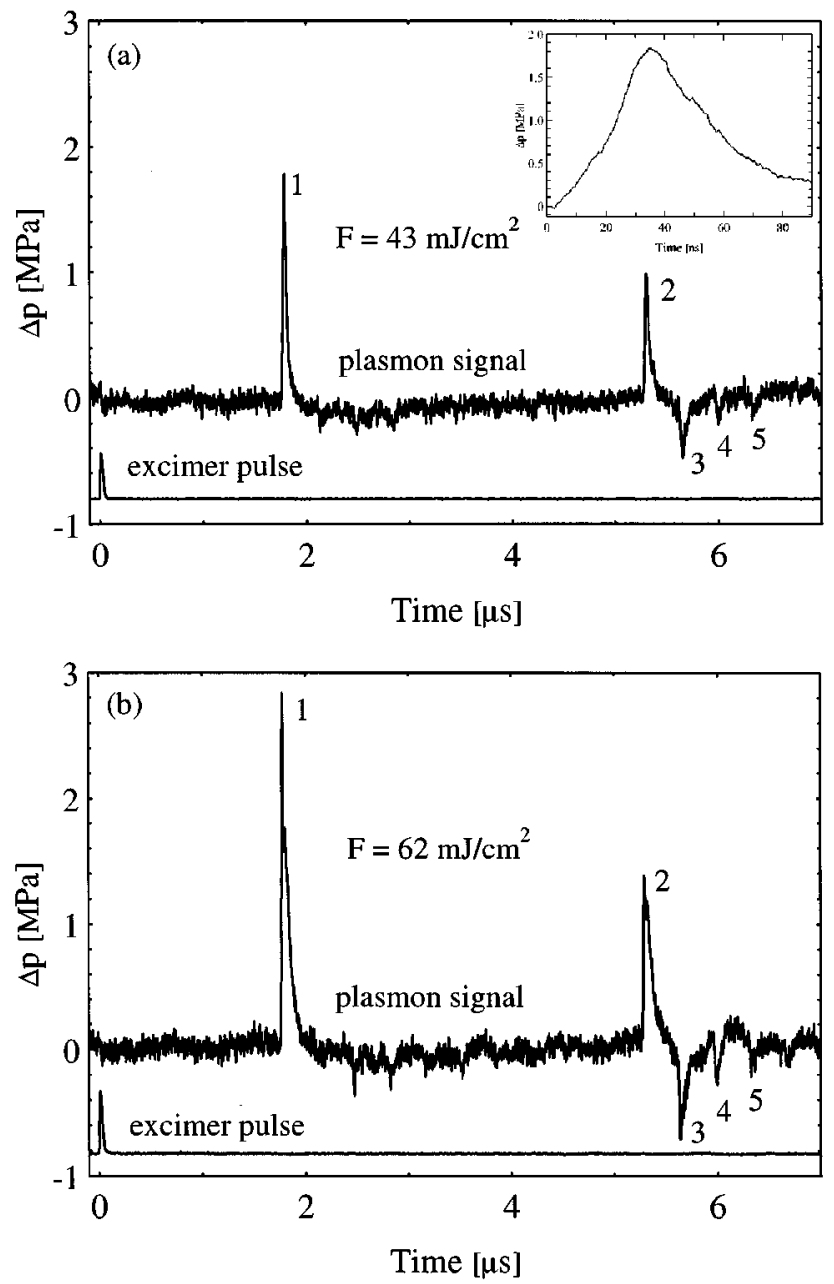

FIG. 3. Time-resolved surface plasmon signals of the acoustic pulses are shown for two different laser fluences, 43 and $62 \mathrm{~mJ} / \mathrm{cm}^{2}$. Peak 1 represents the first pressure pulse arriving at the detector film, whereas pulse 2 is the first echo, and pulses 3 to 5 are multiple echoes with reversed sign. The inset displays an acoustic pulse on a stretched time scale to show the time resolution of this detection method.

the silver film on the quartz slide from the rear side and creates a bubble-growth-induced acoustic pulse at the front which propagates toward the prism. This pulse appears as peak 1 in the plasmon signal. Subsequently, the pulse is reflected from the silver film of the prism due to the acoustic impedance mismatch, and propagates back to the quartz slide. Here part of the pulse is reflected again and detected as the first echo in the plasmon signal (peak 2), whereas the other part of the pulse is transmitted into the quartz slide. After propagation in the quartz slide, this part is partially reflected with a phase change at the quartz-water interface because water is acoustically less dense than quartz glass. The pulse then propagates once more through quartz and the water and is detected as a tensile pulse in the plasmon signal (peak 3). The peaks 4 and 5 are echo signals from multiple reflections in the quartz slide. The verification that the different peaks in the plasmon signal correspond to the specified pulses and echoes was done using quartz slides with different thicknesses which were placed at various distances in front of the glass prism. The quantitative analysis of the time-resolved pressure measurements yielded 1.8 MPa for the maximum pressure in the first pulse for a laser fluence of 
$43 \mathrm{~mJ} / \mathrm{cm}^{2}$ and $2.8 \mathrm{MPa}$ for a fluence of $62 \mathrm{~mJ} / \mathrm{cm}^{2}$.

In order to demonstrate the time resolution of the surface plasmon technique, the first peak of the lower part of Fig. 3 is presented as the inset on an expanded time scale. The temporal profile of the acoustic pulses is dominated by the growth dynamics of the laser-induced gas bubbles. Since the temporal resolution of the setup used is better than a nanosecond, the observed rise time of the plasmon signal, approximately $30 \mathrm{~ns}$ as displayed in the inset of Fig. 3, corresponds to the time the bubbles need to grow to their maximum size. Since the static control experiment was done at an equilibrium temperature, but the compression in the transient acoustic pulses is adiabatic, one has to estimate the temperature rise because the refractive index of water also changes with temperature. Using data tables for water ${ }^{16}{ }^{\text {one }}$ finds a temperature rise of about $0.014{ }^{\circ} \mathrm{C}$ for a pressure increase of $1 \mathrm{MPa}$. This means that the temperature rise due to adiabatic compression in the acoustic pulses can be neglected for our experiments. It should be pointed out that the surface plasmons monitor the exact form of the acoustic pulses and do not average since the decay length of the evanescent field of the surface plasmons (approximately 230 $\mathrm{nm}$ ) in water is small compared to the wavelength of the acoustic pulse (approximately $100 \mu \mathrm{m}$ ). The noise in the signal in Fig. 3, which originates from intermode beating of the $\mathrm{He}-\mathrm{Ne}$ probe laser, presently limits the resolution of the pressure measurements to about $0.1-0.2 \mathrm{MPa}$. With the use of a single mode laser, it should be possible to improve the resolution further.

The results presented in this letter show that surface plasmons can successfully be used for absolute pressure measurements on very short time scales. The method is ca- pable of detecting exact pulse profiles of acoustic waves. The spatial resolution of this technique is only limited by the finite diameter of the probe beam, which was about $1 \mathrm{~mm}$ in the examples shown here. By focusing the probe beam, the method can be extended to obtain spatial resolution in the $\mu \mathrm{m}$ range.

We thank S. Herminghaus for helpful discussions and enlightening ideas. The work was supported by the Center of Modern Optics at the University of Konstanz, Germany.

${ }^{1}$ J. A. Parrish and T. F. Deutsch, IEEE J. Quantum Electron. 20, 1386 (1984).

${ }^{2}$ H. K. Park, C. P. Grigoropoulos, W. P. Leung, and A. C. Tam, IEEE Trans. Compon. Packaging Manuf. Technol. Part A 17, 631 (1994).

${ }^{3}$ A. C. Tam, W. P. Leung, W. Zapka, and W. Ziemlich, J. Appl. Phys. 71, 3515 (1992).

${ }^{4}$ W. Zapka, W. Ziemlich, and A. C. Tam, Appl. Phys. Lett. 58, 2217 (1991)

${ }^{5}$ A. C. Tam, N. Do, N. Klees, P. T. Leung, and W. P. Leung, Opt. Lett. 17, 1809 (1992)

${ }^{6}$ O. Yavaş, P. Leiderer, H. K. Park, C. P. Grigoropoulos, C. C. Poon, N. Do, and A. C. Tam, Phys. Rev. Lett. 70, 1830 (1993).

${ }^{7}$ O. Yavaş, P. Leiderer, H. K. Park, C. P. Grigoropoulos, C. C. Poon, W. P. Leung, N. Do, and A. C. Tam, Appl. Phys. A 58, 407 (1994).

${ }^{8}$ H. K. Park, D. Kim, C. Grigoropoulos, and A. C. Tam, J. Appl. Phys. 80, 4072 (1996).

${ }^{9}$ B. Sullivan and A. C. Tam, J. Acoust. Soc. Am. 75, 437 (1984).

${ }^{10}$ S. Puderbach, S. Herminghaus, and P. Leiderer, Phys. Lett. A 130, 401 (1988).

${ }^{11} \mathrm{~S}$. Herminghaus and P. Leiderer, Appl. Phys. A 51, 350 (1990).

${ }^{12}$ S. Herminghaus and P. Leiderer, Appl. Phys. Lett. 58, 352 (1991).

${ }^{13}$ Schott Glas-Katalog, Germany 1994.

${ }^{14}$ E. Kretschmann and H. Raether, Z. Naturforsch. Teil A 23, 2135 (1968).

${ }^{15}$ P. Schiebener, J. Straub, J. M. H. Levelt Sengers, and J. S. Gallagher, J. Phys. Chem. reference data, 19, 677 (1990).

${ }^{16}$ E. Schmidt, Properties of Water and Steam in SI-Units (Springer, New York, 1979). 